\title{
Una línea de referencia histórica acerca de la distribución espacial del machuelo (Opisthonema oglinum) en el área de afloramiento del mar Caribe de Colombia A historical reference line about the spatial distribution of Atlantic thread herring
(Opisthonema oglinum) in the upwelling area of the Colombian Caribbean Sea
}

\author{
Luis M. Manjarrés-Martínez* ${ }^{\circledR}$ y Félix Cuello ${ }^{\circledR}$ \\ Grupo de Investigación "Evaluación y Ecología Pesquera", Facultad de Ingeniería, Universidad del Magdalena, Santa Marta, Colombia \\ *Autor de correspondencia: Imanjmart@gmail.com \\ Recibido: 14 agosto de 2019 \\ Aceptado: 18 de diciembre de 2019 \\ Publicación en línea: 31 de diciembre de 2019
}

\begin{abstract}
Resumen
Palabras clave:

peces pelágicos; pesca; acústica;

A partir de las densidades de biomasa de peces pelágicos pequeños y los valores de temperatura y geoestadística; mar Caribe

salinidad registrados en tres campañas acústicas efectuadas en el área norte del mar Caribe de Colombia durante el periodo 1997-1998, se llevó a cabo un análisis geoestadístico para describir y modelar la estructura espacial de la densidad de biomasa de machuelo (Opisthonema oglinum), con miras a usarla para evaluar la distribución en toda el área de interés. La comparación de la distribución espacial de 0 . oglinum con la cobertura geográfica de las pesquerías artesanales que explotan esta especie, bien como recurso objetivo o como pesca incidental, muestra que existe una amplia zona de distribución de estos peces a la que no accede ninguna pesquería orientada específicamente a su explotación. De esta manera, se proporciona a la comunidad científica, a las entidades encargadas de la administración pesquera y a los potenciales usuarios de estas especies información orientadora para la explotación y el manejo racional de los peces pelágicos pequeños, en función de la distribución de estos recursos, de las pesquerías existentes y de las variables abióticas relacionadas.
\end{abstract}

Key words: pelagic fish; fishing, acoustics; geostatistics; Caribbean Sea

\section{Abstract}

A geostatistical analysis was performed to model and evaluate the spatial structure of the small-sized pelagic machuelo (Opisthonema oglinum) in the northern Colombian Caribbean Sea, from biomass densities and temperature and salinity data recorded on three acoustic surveys carried out in this area during the period 1997-1998. The comparison between the distribution of 0 . oglinum and the geographical coverage of the artisanal fisheries that capture it, either as target resource or as bycatch, shows a wide zone where this resource is available but there is no fishery attempting its catch. Scientific community, government agencies and potential users are provided with information about distributions of small-pelagic fish and artisanal fisheries effort, both being needed for appropiate exploitation and management measures. 


\section{Introducción}

A nivel mundial la mayoría de las reservas de recursos están siendo explotadas en su potencial máximo o están siendo sobreexplotadas (Ludwig et al., 1993; FAO, 1997; Jackson et al., 2001; Pauly et al., 2003), y existe una lista creciente de especies costeras y oceánicas amenazadas (Baum et al., 2003; Myers y Worm, 2003). Además, las estadísticas mundiales indican una tendencia significativa de reducción de capturas y del tamaño promedio de los peces extraídos en todos los océanos (Pauly et al., 2002). Esta observación tiene fuertes implicaciones en la perspectiva de uso de los recursos, considerando que se ha demostrado poca evidencia de recuperación de las poblaciones de peces marinos una vez caen a niveles críticos de abundancia (Hutchings, 2000).

En el caso particular del Caribe colombiano, se ha encontrado que los recursos demersales están siendo sometidos a un nivel de presión pesquera que amerita la implementación de medidas de manejo tendientes a su sostenibilidad (Manjarrés et al., 2005a, b, c). En contraste, se ha señalado que los pelágicos pequeños registran los mayores niveles de biomasa en el Caribe colombiano (Viaña et al., 1999; Páramo y Roa, 2003), muy superiores a los niveles de captura de tales recursos en la región (De La Hoz et al., 2017).

Dentro de los recursos de peces pelágicos pequeños de mayor interés comercial en el Caribe colombiano, cabe destacar el machuelo (Opisthonema oglinum), el ojo gordo (Selar crumenophthalmus), la caballeta (Decapterus spp.) y la sardina (Sardinella spp.). El machuelo es un clupeido costero que forma densos cardúmenes cerca de la superficie y se distribuye en las regiones tropicales y subtropicales del Atlántico occidental (Hildebrand, 1963; Smith, 1994), desde el Golfo de Maine (Estados Unidos) hasta Santa Catarina (Brasil) (Fisher, 1978). Este recurso tiene un comportamiento migratorio desde la costa hacia mar abierto, sin alejarse grandes distancias (siempre dentro de la plataforma). Según Valdés y Sotolongo (1983), esta especie se desplaza hacía aguas más claras al incrementarse la turbulencia en las zonas costeras por efecto del incremento en la intensidad del viento. A la fecha no se conocen estudios orientados a establecer la presencia de uno o varios stocks en el área de distribución de este recurso, incluso en el contexto solo del Caribe colombiano.

Para el machuelo capturado en la región Caribe en general. se ha registrado una talla máxima de $380 \mathrm{~mm}$ de longitud total (LT) (Cervigón et al., 1992). Sin embargo, para el área de Santa Marta (Departamento del Magdalena, Colombia) Manjarrés et al. (1993) reportan una talla máxima de $330 \mathrm{~mm}$ LT. Houde et al. (1983) basados en la lectura de marcas en otolitos, calculan que el machuelo alcanza durante el primer año una talla de $108 \mathrm{~mm} \mathrm{LH}$. Su crecimiento es de tipo isométrico y su dieta es principalmente microcrustáceos bentónicos, pero varía según la estación, la etapa reproductiva y la edad (Vega-Cendejas et al., 1997). Ecológicamente, esta especie es importante en la dieta de muchos peces predadores grandes (Finucane y Vaught, 1986). En general, las características biológicas de esta especie definen una estrategia de vida de tipo $r$ (Da Rocha et al., 2015).

A partir de una evaluación acústica efectuada a finales de la década de los 90's, la biomasa de machuelo en el Caribe colombiano continental se estimó en alrededor de 62000 t (Viaña et al., 1999). Las estadísticas más recientes disponibles sobre el desembarco anual de esta especie denotan claramente un muy bajo nivel de aprovechamiento de este recurso. En efecto, a partir de las estadísticas colectadas por el Servicio Estadístico Pesquero Colombiano durante el año 2017 por el Servicio Estadístico Pesquero Colombiano (SEPEC) en el departamento del Magdalena (De La Hoz-M et al., 2017), se ha estimado una captura anual de machuelo de $239 \mathrm{t}$, cifra que significa el 17,1\% del desembarco total artesanal en el Departamento del Magdalena durante el año 2017, estimado en 1401 t (Curiel-Pérez, 2018).

Este bajo nivel de uso de este recurso está relacionado con el hecho de que solo puede ser explotado cuando presenta una distribución netamente costera, en cuyo caso los pescadores artesanales utilizan principalmente chinchorros de jala, un arte de arrastre que es operado desde la playa en ensenadas con escasa pendiente. Este tipo de arte ha sido tradicionalmente empleado por los pescadores artesanales del Caribe colombiano, especialmente en la zona costera del departamento del Magdalena, donde el machuelo es empleado como carnada y también como alimento, supliendo ocasionalmente las necesidades de proteína de sectores poblacionales de bajos recursos. En el resto del Caribe colombiano continental las capturas de esta especie tiene un carácter incidental. Otro factor determinante en la sobreexplotación de este recurso es el hecho de que a la fecha no se ha desarrollado una pesquería con capacidad de explotar estos recursos mar afuera, utilizando un arte activa.

El análisis anterior sugiere la posibilidad estratégica de incrementar el esfuerzo pesquero orientado a la captura 
de estos recursos en la región, lo que implica analizar la viabilidad de expandir la actual frontera pesquera de las pesquerías costeras que explotan estos recursos. Además, surge la necesidad de ahondar en el conocimiento científico sobre su distribución y los aspectos biológicos y las características ambientales asociadas a dicha distribución. Por esta razón, a pesar del tiempo transcurrido se considera relevante proporcionar a la comunidad científica, a las entidades encargadas de la administración pesquera y a los potenciales usuarios de estos recursos un conocimiento histórico que constituye una línea base para referenciar posibles cambios tanto en la distribución como en las biomasas de los principales recursos de preces pelágicos pequeños en el área de afloramiento del mar Caribe de Colombia (MCC). Esto se considera particularmente relevante, tomando en consideración que la pesquería artesanal se ve cada vez más reducida a una pesquería de subsistencia y la industria pesquera presenta una flota de embarcaciones sobredimensionada, dados los actuales recursos objetivos. Por tanto, la explotación de estos recursos a un nivel industrial se constituye en una alternativa socio-económica para la región (Duarte y García, 2003).

Naturalmente, para garantizar la sostenibilidad de una pesquería con mayor poder de pesca de estos recursos, es recomendable adoptar medidas de manejo espacialmente explicitas. Para ello, se requiere colocar a las especies explotadas en el contexto del ecosistema donde existen, del cual dependen y con el cual interactúan, si se desea reducir la incertidumbre asociada a la trayectoria espacio-temporal de sus biomasas y de sus estructuras demográficas (Pitcher, 1998). Se ha argumentado que la falta de consideraciones integrales desde el inicio del desarrollo de las pesquerías constituye una de las principales causas del deterioro de los recursos. Por tanto, el diseño de modelos poblacionales y ecosistémicos y las estrategias de aprovechamiento deben considerar la distribución de los recursos para optimizar sus rendimientos en un esquema de uso responsable (Dunning et al., 1995; Walters et al., 1999; Duarte y García, 2001).

El reto del análisis de las poblaciones objetivo de la pesquería a la escala del ecosistema implica considerar los patrones y la heterogeneidad espacial como determinantes de la variabilidad temporal, así como la interacción de la selección natural y los mecanismos de retroalimentación internos que determinan su dinámica (O’Neill 2001). Es así que la comunidad científica envuelta en el estudio de recursos pesqueros ha reconocido que las aproximaciones que busquen contribuir al desarrollo y manejo de recursos marinos vivos deben tener un carácter integral que involucre las dimensiones espaciales y temporales de su variabilidad (Mangel et al., 1996). En efecto, en las últimas décadas se ha hecho énfasis en la importancia de considerar los patrones espaciales de las poblaciones explotadas, toda vez que su dinámica poblacional, las interacciones intra e interespecíficas, los forzantes abióticos y la presión pesquera están estructurados espacialmente (Summers y Rose, 1987; Holmes et al., 1994; Dunning et al., 1995).

En consideración a lo anteriormente expuesto, en el presente trabajo se hace uso de dos herramientas de utilidad para el manejo espacial: sistemas de información geográfica (SIG) y geoestadística. En sistemas tropicales de alta diversidad el SIG puede ser la única herramienta de manejo disponible (Sumaila et al., 2000). Como se ha observado en otros campos de la ciencia en los cuales ocurren problemas relacionados con el espacio, el uso de SIG se puede traducir en un notable mejoramiento del manejo espacial, particularmente cuando se combina con otras herramientas analíticas y modelos (Meaden y Do Chi, 1996). Muchas de las aplicaciones de los SIG están encaminadas a entender fenómenos que tienen una dimensión geográfica, además de una dimensión temporal (e.g. la distribución de un recurso pesquero). Esto permite orientar la asignación de cuotas de pesca y la distribución del esfuerzo a implementar en una pesquería de un recurso (Meaden y Kapetsky, 1992).

A las ventajas de la técnica SIG para la visualización de la información se ha sumado el uso de la geoestadística para una mayor utilidad de la información disponible. Esto implica el uso de un conjunto de métodos diseñados para estudiar variables distribuidas en el espacio, denominadas variables regionalizadas (Rivoirard et al., 2000). De esta manera se pueden obtener mapas de las pesquerías, los recursos y los factores bióticos y abióticos con que interactúan, lo que constituye un insumo prioritario cuando se planea el desarrollo y manejo pesquero (Caddy y García, 1986; Meaden y Do Chi, 1996).

Los datos usados en este estudio corresponden a tres campañas acústicas de evaluación de peces pelágicos pequeños realizadas en el Caribe colombiano por el Programa de Pesca INPA -VECEP/UE con el objetivo general de evaluar los recursos de peces pelágicos pequeños de interés actual o potencial, en diferentes épocas climáticas. Con anterioridad a estas tres campañas, se efectuaron dos exploraciones de este tipo en el área. La primera comprendió una campaña efectuada por el Proyecto Colciencias-CIID-FES en 1985, 
en el que se evaluó la distribución de los recursos y los bio-volúmenes o abundancias relativas, y la segunda abarcó tres campañas realizadas por FAO-NORAD en 1988 (Stromme y Saetersdal, 1989). En lo referente a pelágicos, los objetivos específicos de la evaluación de FAO-NORAD se refirieron a los recursos machuelo (Opisthonema oglinum) y Sardina (Sardinella sp.) en la Península de La Guajira. Los datos acústicos de la campaña INPA-VECEP/UE 9707 fueron objeto de un análisis estadístico por Páramo y Roa (2003), para efectos de estimar la biomasa de las cuatro principales especies de pelágicos pequeños y su distribución espacial, además de explorar las relaciones entre la abundancia de peces y las variables ambientales. Sin embargo, en este trabajo se analizan y comparan los resultados de las tres campañas, en virtud al marcado condicionamiento de la distribución de estos recursos a la variabilidad ambiental.

\section{Aspectos metodológicos \\ Fuentes de información}

Tanto los datos de abundancia de los recursos de peces pelágicos pequeños como los registros de temperatura y salinidad superficiales del mar (TSM y SSM, respectivamente) se obtuvieron mediante tres campañas acústicas efectuadas por el Programa de Pesca INPA-VECEP/UE (Viaña et al., 1999), durante el período 1997-1998, a bordo de un barco de investigación con arrastre por popa, el R/V "Hermano Ginés", de la Fundación La Salle de Venezuela. La campaña PELAG 9707 (julio-agosto de 1997) comprendió un trayecto acústico de $582 \mathrm{mn}$, durante el cual se efectuaron 17 lances de pesca comprobatoria. El trayecto acústico de la PELAG 9711 (octubre-noviembre de 1997) fue de $885 \mathrm{mn}$, recorrido que comprendió un total de 34 lances de pesca, en tanto que el trayecto de la PELAG 9804 (abril de 1998) implicó un barrido acústico de $828 \mathrm{mn}$ y un total de 18 lances de pesca.

En la campaña 9707 no se pudieron efectuar estaciones oceanográficas en el área norte de La Guajira, debido a inconvenientes técnicos. El rastreo acústico se realizó con una ecosonda científica SIMRAD, modelo EK-500, con la cual se hizo un barrido de tipo sistemático, basado en transectas paralelas perpendiculares a la costa, separadas $12 \mathrm{mn}$ en promedio y con longitud variable entre 11 y $25 \mathrm{mn}$, dependiendo de la extensión de la plataforma continental. Las mediciones acústicas se hicieron de acuerdo con los procedimientos estándar. La frecuencia de operación del transductor fue de $38 \mathrm{KHz}$ y la duración del pulso $1 \mathrm{~ms}$. La calibración fue hecha mediante el método de blanco estándar recomendado por ICES (Foote,1987).
Durante el rastreo acústico, realizado entre las $06: 00$ y las 20:00 horas aproximadamente, se prospectó la columna vertical desde los $3,5 \mathrm{~m}$ de profundidad, con un intervalo de $100 \mathrm{~m}$ de amplitud, límite de la supercapa fijada en la configuración del ecosonda. Se establecieron unidades básicas de muestreo (UBM) de 1 $\mathrm{mn}$, para cada una de las cuales se estableció un valor de ecointegración (Sa), también conocido como coeficiente de retrodispersión sonora por unidad de área o valor promedio de la intensidad de ecos por área, dado en $\mathrm{m}^{2} / \mathrm{mn}^{2}$. Este valor se usó como un índice acústico de la abundancia multiespecífica relativa de recursos de peces pelágicos.

\section{Construcción de los mapas temáticos SIG}

Las densidades de biomasa de peces pelágicos pequeños y los valores de TSM y SSM correspondientes a cada una de las tres campañas acústicas efectuadas por el programa de pesca INPA-VECEP/UE (Viaña et al., 1999), fueron consignados en mapas temáticos SIG, los cuales fueron digitalizados a partir de las cartas náuticas COL 040 (Bocas de Ceniza a Riohacha) y COL 041 (Riohacha a Punta Espada), publicadas por la Dirección General Marítima y Portuaria (DIMAR). Para georreferenciar las densidades de peces, se utilizó la posición exacta de la finalización de cada UBM (unidad básica de muestreo) y para la TSM y SSM la posición de cada estación oceanográfica. Además de los mapas de densidad por UBM, se utilizó el análisis geoestadístico para interpolar gráficamente los datos de densidad de cada recurso y de las variables oceanográficas.

Dada la condición no aleatoria del muestreo acústico, se usó estadística intrínseca para describir y modelar la estructura espacial de la densidad de biomasa y su varianza para todas las especies evaluadas en el estudio, a través de estimadores insesgados de modelo (Páramo y Roa, 2003). Esto implicó el uso de variogramas como herramienta estructural básica (Clark, 1979; Rivoirard et al., 2000). Se incluyeron las intertransectas por cuanto Rivoirard et al. (2000) encontraron que la exclusión de éstas en un muestreo de tipo sistemático con transectas paralelas e intertransectas no desmejora la estabilidad de los variogramas. El análisis geoestadístico se llevó a cabo mediante el software GS+. El análisis geoestadístico se inició con la descripción univariada de $\rho$, a través de histogramas de frecuencias, para evaluar simetría (Isaaks y Srivastava, 1989). Posteriormente, se construyeron gráficos tipo posting, para detectar la ocurrencia de valores extremadamente altos o bajos, lo que podría indicar cambios espaciales en la variabilidad local. Una relación entre la variabilidad local y la media 
local denota una distribución lognormal de los datos, situación usual en datos de densidad de peces obtenidos mediante ecointegración (Rivoirard et al., 2000; Páramo y Roa, 2003). En tales casos, para reducir el efecto de los valores extremos se realizó una transformación logarítmica de los datos (Isaaks y Srivastava, 1989; Petitgas y Prampart, 1993; Goovaerts, 1997; Rivoirard et al., 2000). También se construyeron diagramas de dispersión-h ( $\mathrm{h}$-scatterplots) para identificar puntos atípicos y chequear la conveniencia de removerlos. Para las nubes de puntos correspondientes a diferentes valores de $\mathrm{h}$ y diferentes direcciones se calculó el respectivo coeficiente de correlación. A partir de estos coeficientes de correlación se construyeron los correlogramas (Isaaks y Srivastava, 1989).

El proceso de modelación incluyó tres fuentes de información: 1) semivariograma experimental, 2) semivariograma permisible y 3) información auxiliar (uso de medidas robustas como el madograma para evaluar direcciones de anisotropía, proporción y rango) (Goovaerts, 1997). Para detectar anisotropía se usó el enfoque convencional de comparar semivariogramas experimentales calculados en varias direcciones, complementado con un mapa de semivariograma (Goovaerts, 1997). Para los semivariogramas experimentales se usaron los ángulos de $0^{\circ}, 45^{\circ}, 90^{\circ} \mathrm{y}$ $135^{\circ}$ con un ángulo de tolerancia $\Delta \theta= \pm 22,5^{\circ}$. Al igual que Páramo y Roa (2003), no se encontró efecto anisotrópico en los variogramas experimentales.

Para evaluar los parámetros del kriging se usó la validación cruzada (Páramo y Roa, 2003). Este método consiste en remover un dato cada vez del conjunto de datos y reestimar este valor a partir de los datos remanentes usando los diferentes modelos de semivariograma. Se comparan los datos reales con los interpolados y el modelo que produce las predicciones más precisas es seleccionado (Gamma Design Software, 2004). La vecindad de búsqueda en el kriging corresponderá a la extensión hasta la cual se plantea la hipótesis de estructura espacial (estacionalidad local) (Rivoirard et al., 2000).

\section{Resultados}

\section{Densidades de biomasa de peces pelágicos}

Se ajustaron diferentes tipos de modelos de variogramas isotrópicos omnidireccionales a los datos de densidad de biomasa de machuelo de las tres campañas científicas (tabla 1). Incluso para la campaña 9797 se ajustaron dos modelos diferentes: uno para la zona norte (esférico) y otro para la zona sur (exponencial). Los valores del coeficiente de determinación evidenciaron ajustes aceptables para los diferentes modelos, destacándose especialmente los valores correspondientes a la 9707 (zona sur) y a la 9711, que superaron el $85 \%$. En los restantes variogramas se obtuvieron valores de $R^{2}$ superiores al $75 \%$.

A partir de los modelos de variogramas ajustados se generaron los respectivos mapas temáticos de densidades de biomasa, los cuales muestran una característica común: cambios temporales en la distribución y abundancia. Durante la campaña PELAG 9707 (época de calmas), la especie presentó una distribución costera y extendida a todo lo largo del área prospectada, exceptuando los sectores frente a la Ciénaga Grande de Santa Marta (CGSM) y entre Riohacha y Manaure. Las mayores concentraciones (hasta 15,2 t/mn ${ }^{2}$ ) se observaron a la altura de Bahía Portete (entre Punta Cañón y Punta Gallinas). Se presentaron concentraciones intermedias entre Palomino y Riohacha (hasta $12,2 \mathrm{t} / \mathrm{mn}^{2}$ ), a lo ancho de toda la plataforma, y entre Manaure y Punta Cañón (Bahía Honda), y las concentraciones bajas ocurrieron en la zona de Santa Marta (figura 1a). Esta especie fue capturada en el $45 \%$ de los lances de pesca con red de arrastre pelágico. Las mayores extracciones se produjeron en horas de la noche y al amanecer (18:00 a 05:00), a menos de $10 \mathrm{mn}$ de la costa y a profundidades de pesca inferiores a $40 \mathrm{~m}$.

Tabla 1. Parámetros y criterios de bondad de ajuste de los modelos ajustados a variogramas clásicos isotrópicos omnidireccionales para la densidad de biomasa de las especies evaluadas en las tres campañas científicas. RSS=suma de cuadrados reducida para ajustar numéricamente los modelos de variogramas.

\begin{tabular}{rlrrrrrr}
\hline Campaña/ Zona & Modelo & $\begin{array}{c}\text { Efecto Pepita } \\
\text { C0 }\end{array}$ & $\begin{array}{c}\text { Meseta } \\
\text { C0 }+\mathbf{C}\end{array}$ & Rango & RSS & R $^{\mathbf{2}}$ & \multirow{2}{*}{ C/(Co+C) } \\
\hline $9707 /$ Zona norte & Esférico & 0,0379 & 0,1368 & 12,9 & 0,0025 & 0,771 & 0,723 \\
$9707 /$ Zona sur & Exponencial & 0,0840 & 1,1710 & 8,7 & 0,1080 & 0,881 & 0,928 \\
9711 & Gaussiano & 0,1900 & 0,9630 & 10,2 & 0,1160 & 0,875 & 0,803 \\
9804 & Exponencial & 0,2395 & 0,5210 & 6,3 & 0,0113 & 0,754 & 0,540 \\
\hline
\end{tabular}


Durante la campaña PELAG 9707 (época de calmas) la especie presentó una distribución costera y extendida a todo lo largo del área prospectada, exceptuando los sectores frente a la Ciénaga Grande de Santa Marta (CGSM) y entre Riohacha y Manaure. Las mayores concentraciones (hasta 15,2 t/mn ${ }^{2}$ ) se observaron a la altura de Bahía Portete (entre Punta Cañón y Punta Gallinas). Se presentaron concentraciones intermedias entre Palomino y Riohacha (hasta 12,2 t/mn ${ }^{2}$ ), a lo ancho de toda la plataforma y entre Manaure y Punta Cañón (Bahía Honda).

Concentraciones bajas ocurrieron en la zona de Santa Marta (figura 1a). Esta especie fue capturada en el $45 \%$ de los lances de pesca con red de arrastre pelágico. Las mayores capturas se produjeron en horas de la noche y al amanecer, a menos de $10 \mathrm{mn}$ de la costa y a profundidades de pesca inferiores a $40 \mathrm{~m}$.
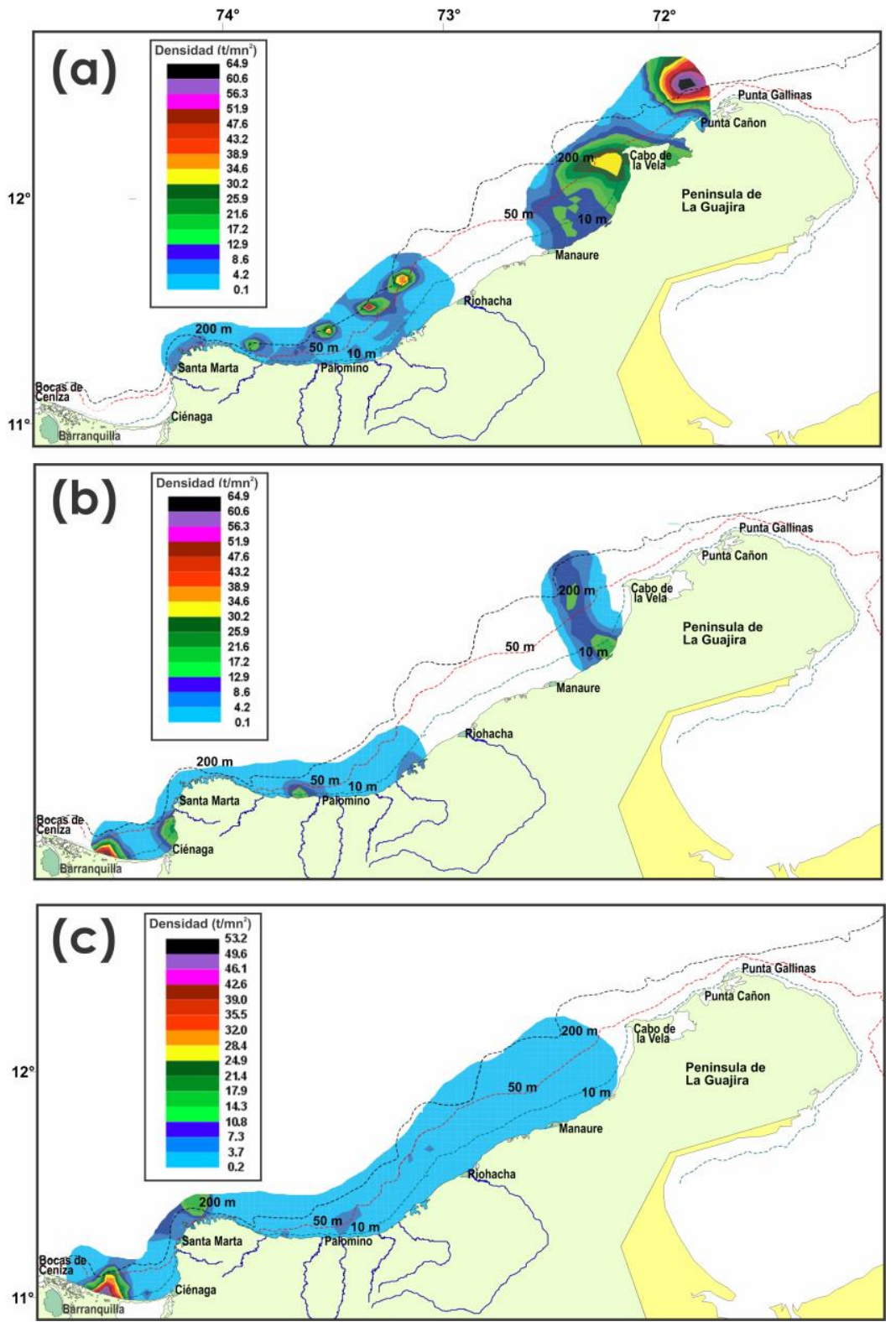

Figura 1. Distribución de la densidad de biomasa de machuelo (Opisthonema oglinum): a) campaña 9707, b) campaña 9711, c) campaña 9804.

En contraste con lo ocurrido en la campaña PELAG 9707, durante la 9711 (final de época de calmas) y la 9804 (final de época de vientos) se registraron altas

153
En esta zona la densidad llegó a 21,6 t/mn² durante el 9711 (figura 1b). En general, en esta campaña el recurso tuvo una distribución más restringida, pero se presentaron mayores densidades. 
Además de la zona ya mencionada, se registraron concentraciones altas entre Manaure y el Cabo de la Vela. A la altura de Palomino y de Bahía Portete se registraron densidades intermedias. Esta especie fue capturada en el intervalo de profundidades de 12 (Golfo de Salamanca) a $98 \mathrm{~m}$ (frente a Camarones), a diferentes horas del día, entre 1 y $8 \mathrm{mn}$ de la costa.

Una característica notoria en el PELAG 9804 fue la ausencia del recurso en la zona ubicada al norte del Cabo de la Vela y su amplia distribución en el resto del área evaluada, con tendencia a aumentar las densidades en dirección NE-SO (figura 1c). En esta campaña la distribución del machuelo fue más continua que en las dos campañas anteriores, pero con un mayor predominio de densidades bajas, especialmente en la plataforma Guajira, donde las densidades medias no excedieron de $7,3 \mathrm{t} / \mathrm{mn}^{2}$. Las mayores concentraciones (hasta 53,2 t/mn2) se presentaron cerca de la desembocadura del Río Magdalena $\left(75-80 \mathrm{t} / \mathrm{mn}^{2}\right)$ y en el área del Parque Nacional Natural Tayrona, cerca de Santa Marta (hasta 17,9 t/mn²).

Tabla 2. Estadísticos descriptivos de los datos registrados en las estaciones oceanográficas realizadas en las tres campañas de investigación.

\begin{tabular}{llcccccccc}
\hline \multirow{2}{*}{ Campaña } & \multirow{n}{*}{$\mathbf{n}$} & \multicolumn{4}{c}{ Temperatura superficial } & \multicolumn{3}{c}{ Salinidad superficial } \\
& & Media & S & Min & Máx & Media & S & Min & Máx \\
\hline 9707 & 40 & 26,49 & 0,90 & 24,96 & 28,67 & 36,67 & 0,41 & 35,62 & 37,78 \\
9711 & 43 & 26,34 & 1,44 & 23,12 & 28,51 & 35,26 & 3,90 & 17,05 & 37,45 \\
9804 & 38 & 26,79 & 1,03 & 25,27 & 28,99 & 36,41 & 1,58 & 27,04 & 37,03 \\
\hline
\end{tabular}

Tabla 3. Parámetros, criterios de bondad de ajuste y validación cruzada de los modelos ajustados a variogramas clásicos isotrópicos omnidireccionales para la TSM. RSS= suma de cuadrados reducida para ajustar numéricamente los modelos de variograma; $\mathrm{RC}=$ coeficiente de bondad de ajuste por mínimos cuadrados de la ecuación de regresión lineal respecto a la línea de $45^{\circ}, E E P R E D=$ error estándar de las predicciones.

\begin{tabular}{lcclcccccc}
\hline Campaña & Lag & $\begin{array}{c}\text { Tolerancia de } \\
\text { Lag }\end{array}$ & Modelo & $\begin{array}{c}\text { Efecto } \\
\text { pepita C0 }\end{array}$ & $\begin{array}{c}\text { Meseta } \\
\text { C0+C1 }\end{array}$ & Rango & RSS & \multicolumn{2}{c}{ Validación cruzada } \\
RC & EE PRED. \\
\hline 9707 & 0,25 & 0,12 & Esférico & 0,371 & 0,905 & 1,120 & 0,0316 & 0,927 & 0,777 \\
9711 & 0,20 & 0,10 & Gaussiano & 0,190 & 3,288 & 1,282 & 0,0162 & 0,991 & 0,577 \\
9804 & 0,20 & 0,10 & Esférico & 0,133 & 1,870 & 2,150 & 0,0530 & 0,998 & 0,672 \\
\hline
\end{tabular}

\section{Análisis espacial de la temperatura y la salinidad}

Los promedios de temperatura fueron similares entre campañas, pero la campaña 9711 tuvo una mayor dispersión en los datos. No se detectó relación entre las medias locales y las desviaciones estándar locales (tabla 2). Se determinaron los variogramas empíricos omnidireccionales que mejor describieron la continuidad espacial de los datos oceanográficos. En el caso de la TSM, se ajustó un modelo esférico para las campañas 9797 y 9804, y un modelo gaussiano para la campaña 9711 (tabla 3). En la exploración de anisotropia, se observó en todos los casos una superficie de características isotrópicas, es decir, se obtuvieron valores muy similares de semivarianza promedio para diferentes direcciones en el mapa de variogramas.

La distribución de la TSM en la campaña 9707 indica la presencia de un núcleo de afloramiento fuerte en el norte de la Península de La Guajira con temperaturas por debajo de los $26{ }^{\circ} \mathrm{C}$ y un núcleo de afloramiento débil en inmediaciones de Santa Marta con temperaturas entre $26^{\circ} \mathrm{C}$ y $27^{\circ} \mathrm{C}$. En el sur de La Guajira $y$ en el sector del PNNT se registró una zona de aguas más cálidas (figura 2a). En la campaña 9711 se observó un aumento de la TSM en sentido nororientesuroccidente, como consecuencia de la permanencia de un núcleo de afloramiento en el norte de la Península de La Guajira y aumento de temperatura en el golfo de Salamanca. El sector del sur de La Guajira nuevamente presentó una zona de aguas cálidas (figura 2b). La TSM registrada en la campaña 9804 evidenció la presencia del evento del afloramiento en toda la zona evaluada, además de un área de aguas cálidas en el sector comprendido entre el sur de La Guajira y el PNNT (figura 2c). 

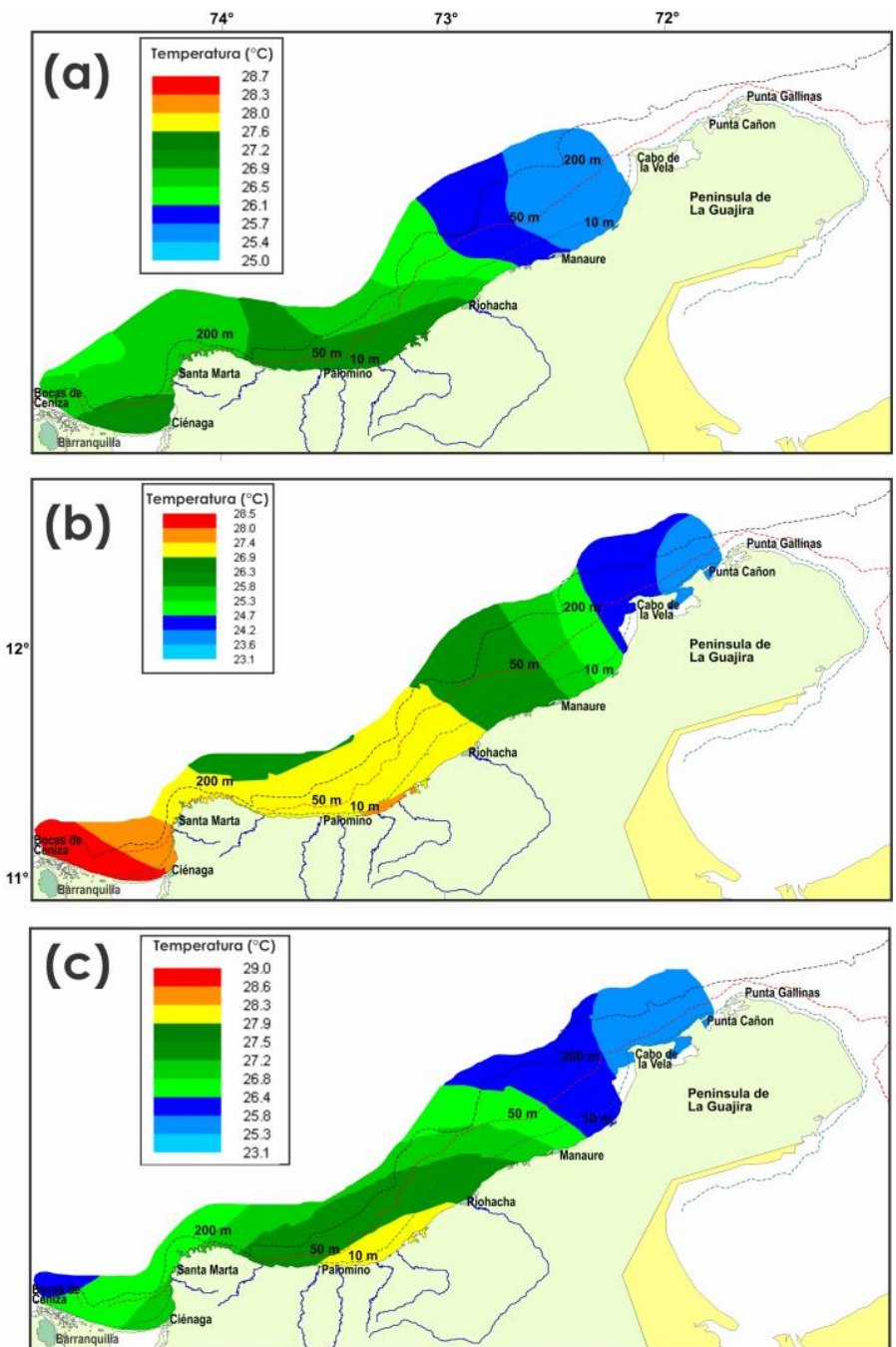

Figura 2. Distribución espacial de la temperatura superficial del mar (TSM), obtenida mediante kriging ordinario. a) campaña 9707, b) campaña 9711 y c) campaña 9804.

En la campaña 9707 los menores valores de SSM $(<36,4)$ se registraron en la zona del PNNT y los mayores $(>36,8)$ en la franja costera ubicada entre Camarones y Manaure. En el resto del área evaluada la salinidad registró valores intermedios, entre 36,4 y 36,8 (figura 3a). En la campaña 9711 se observó un aumento de la SSM en sentido suroccidente-nororiente, con valores de SSM menores de 35 en la zona del golfo de Salamanca y mayores de este valor en aguas de la Península de La Guajira, a excepción de las estaciones más oceánicas situadas frente al sector Cabo de la VelaPunta Gallinas, donde se registraron salinidades menores de 36,4 (figura 3b). Análogamente a lo comentado respecto a la distribución espacial de la TSM, el comportamiento de la SSM durante la campaña 9804 puso de presente la influencia del afloramiento estacional que tiene su núcleo en el sector la parte alta de la península guajira. En esta zona la SSM alcanzó valores que fluctuaron entre 36,6 y 37,0 , en tanto que valores que fluctuaron entre 36,0 y 36,4 (figura 3c).

\section{Discusión}

Una de las características más notorias en la distribución de la TSM y la SSM en el área de afloramiento del mar Caribe de Colombia es la presencia de una zona de aguas cálidas ubicada entre el sur de la Península de La Guajira y el PNNT. Las características de esta zona parecen corresponder a un área donde se verifican los procesos de enriquecimiento, concentración y retención que se han propuesto como necesarios en regiones de afloramiento para el desarrollo de poblaciones pelágicas, particularmente en sus estadios tempranos (Bakun, 1996). En general, se detectaron procesos de afloramiento en el sector norte durante todos los meses Esto corrobora la presencia de afloramiento a lo largo del año en el área de estudio, aunque con intensidad variable de acuerdo al patrón estacional de los vientos alisios en la región (Andrade y Barton, 2005). 

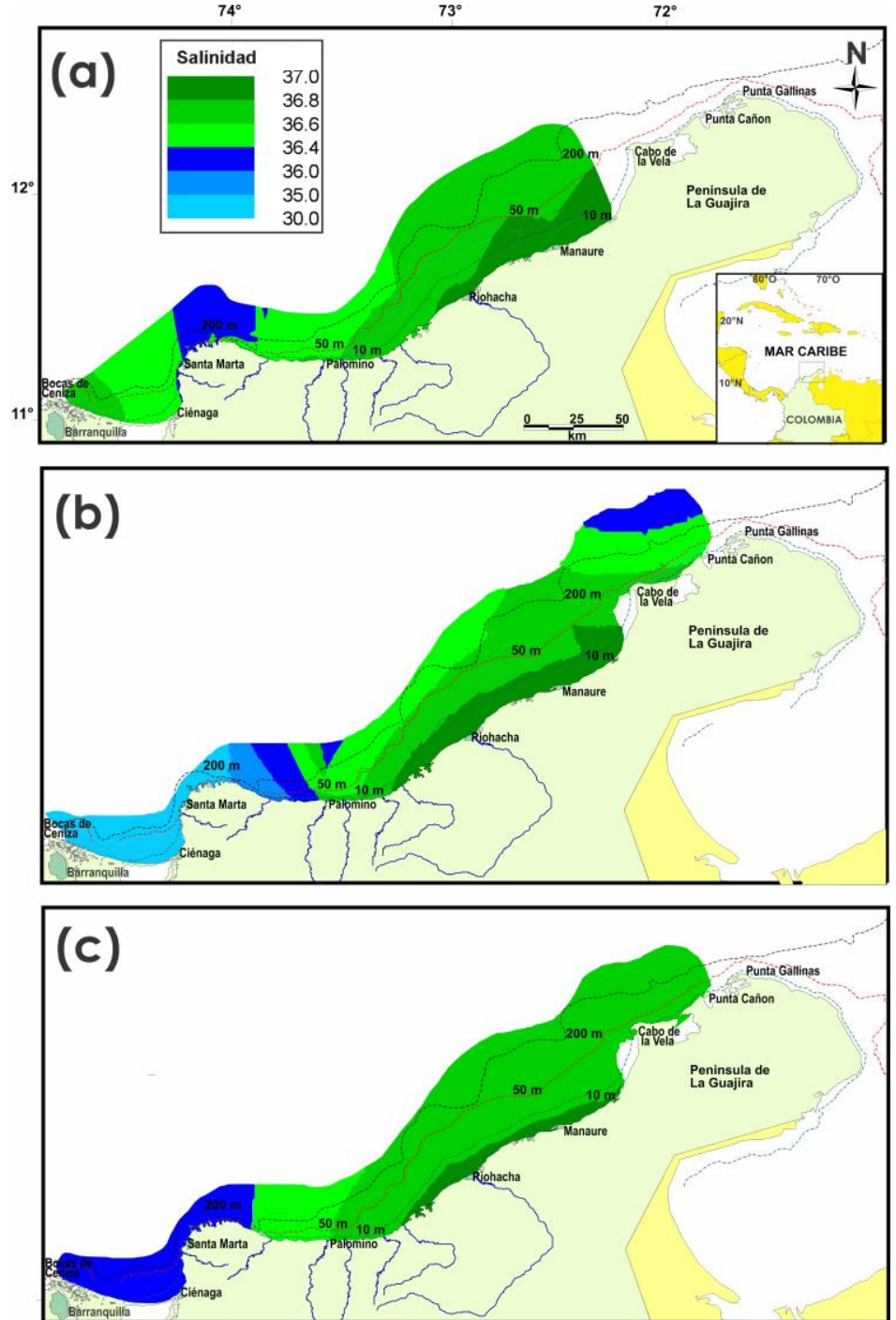

Figura 3. Distribución espacial de la salinidad superficial, obtenida mediante kriging ordinario. a) campaña 9707, b) campaña 9711 y c) campaña 9804.

Aunque el machuelo (Opisthonema oglinum) tuvo una distribución amplia, en las tres campañas las mayores abundancias de esta especie estuvieron asociadas a la zona de aguas cálidas descrita previamente, lo cual reafirma la hipótesis de la idoneidad de dicha zona para el desarrollo de la población, desde la misma fase larval (Kemmerer, 1977; Viaña et al., 1999). En la campaña 9804 las mayores densidades de machuelo estuvieron asociadas al intervalo de temperatura superficial 27 $28,5{ }^{\circ} \mathrm{C}$, en un rango de salinidades relativamente amplio (35 - 37). Estos resultados guardan relación con la apreciación de Kinnear y Fuss (1971) (citado por Finucane y Vaught, 1986), en el sentido de que el pico de adultos de esta especie en el golfo de México se encuentra entre 26 y $29^{\circ} \mathrm{C}$. Según Kenmerer (1977), en el Golfo de México el machuelo prefiere aguas calientes, entre 28,5 y $29,8{ }^{\circ} \mathrm{C}$. En este sentido, cabe resaltar que aunque en la campaña 9804 se hizo un menor número de lances de pesca, se evidenció un ajuste aceptable del variograma a los datos observados.

En lo que respecta a la comparación entre las disponibilidades de recursos de peces pelágicos pequeños determinadas mediante campañas científicas y los rendimientos de las pesquerías artesanales que explotan estos recursos en una cobertura geográfica restringida a la zona costera del departamento del Magdalena, es evidente que existen posibilidades de expandir esta pesquería tanto geográficamente como en términos de los rendimientos pesqueros (Barros y Manjarrés, et al., 2004; De La Hoz-M et al., 2017). El estudio pone de presente que en el área norte del mar Caribe de Colombia existe una amplia zona de distribución de estos recursos que no es accedida por 
ninguna pesquería orientada específicamente a su explotación. Lo anterior reafirma el resultado de Viaña et al. (1999), quienes estimaron biomasas relativamente altas de peces pelágicos pequeños, en comparación con sus respectivos niveles de explotación.

En la actualidad, la vulnerabilidad de este recurso es muy dependiente de los movimientos de sus cardúmenes hacía la costa, donde opera la pesquería que usualmente lo explota (Vega-Cendejas et al., 1997). En lo que respecta específicamente al Caribe colombiano, a la fecha la captura del machuelo en calidad de recurso objetivo sigue siendo exclusiva de la pesquería artesanal de chinchorros de jala que opera en la zona de Santa Marta (De La Hoz-M et al., 2017), Por tanto, para desarrollar una pesquería sostenible de este recurso en aguas abiertas deben superarse aún algunos inconvenientes de tipo tecnológico, tendientes a posibilitar una pesca activa del mismo, particularmente mediante el uso de redes cerco (Manjarrés et al., 1993; Altamar y Zúñiga, 2015). De esta manera, mediante la integración de herramientas de SIG y de técnicas de geoestadística, el presente trabajo proporciona a la comunidad científica, a las entidades encargadas de la administración pesquera y a los potenciales usuarios de estos recursos una información de interés para monitorear el posible impacto del desarrollo de una pesquería de este tipo y de las medidas de manejo que se adopten para un uso responsable de este recurso en el ecosistema de afloramiento del mar Caribe de Colombia.

\section{Referencias}

Altamar, J. y Zúñiga, H. 2015. Cuantificación de unidades económicas de pesca y caracterización de artes y embarcaciones de pesca artesanales en Colombia. Autoridad Nacional de Acuicultura y Pesca (AUNAP), Bogotá.

Andrade, C. y Barton, E.D. 2005. The Guajira upwelling system. Continental Shelf Research 25:1003-1022. Doi: https://doi.org/10.1016/j.csr.2004.12.012.

Bakun, A. 1996. Patterns in the Ocean. California Sea Grant College System. NOAA. Centro de Investigaciones Biológicas del Nordeste, La Paz, México.

Barros, M. y Manjarrés, L. 2004. Recursos pesqueros explotados por las pesquerías artesanales marítimas del sector Taganga-La Jorará (Dpto. del Magdalena), con énfasis en peces demersales. En: Manjarrés, L. Editor. Pesquerías demersales del área norte del Mar Caribe de Colombia y parámetros ecológicos y poblacionales del recurso pargo. Universidad del Magdalena, Santa Marta.
Baum, J.K., Myers, R.A., Kehler, D.G., Worm, B., Harley, S.J. y Doherty, P.A. 2003. Collapse and Conservation of Shark Populations in the Northwest Atlantic. Science 299: 389392.

Caddy, J.F. y García, S.M. 1986. Fisheries thematic mapping - A prerequisite for intelligent management and development of fisheries. Oceanographie Tropicale 21(1): 31-52.

Cervigón, F.; Cipriani R.; Fischer W.; Garibaldi, L.; Hendrickx M., Lemus, A.J.; Márquez, R.; Poutiers, J.M.; Robaina, G. y Rodriquez, B.1992. Fichas FAO de Identificación de Especies para los Fines de la Pesca Guía de Campo de las Especies Comerciales Marinas y de Aguas Salobres de la Costa Septentrional de Sur América. Organización de las naciones unidas para la agricultura y la alimentación (fao), Roma.

Clark, I. 1979. Practical Geostatistics. Elsevier Publishing Inc. New York.

Curiel-Pérez, J.E. 2018. Reconstrucción de las estadísticas de desembarco de las pesquerías artesanales marinas del departamento del Magdalena (Caribe colombiano) para los años 1995, 2008 y 2017. Tesis de grado. Ingeniería Pesquera. Universidad del Magdalena, Santa Marta, Colombia.

Da Rocha, D., Franco, M., Gatts, P. y Zalmon, I. 2015. The effect of an artificial reef system on the transient fish assemblages-south-eastern coast of Brazil. Journal of the Marine Biological Association of the United Kingdom 95: 635 - 646 Doi: https://doi.org/10.1017/S0025315414001519.

De la Hoz-M, J., Duarte, L.O. y Manjarrés-Martínez, L. 2017. Estadísticas de desembarco y esfuerzo de las pesquerías artesanales e industriales de Colombia entre marzo y diciembre de 2017. Informe técnico. Autoridad Nacional de Acuicultura y Pesca (AUNAP), Universidad del Magdalena, Santa Marta.

Duarte, L.O. y García, C.B. 2003. Trophic role of small pelagic fishes in a tropical upwelling ecosystem. Ecological Modelling 172: 323-338. Doi: https://doi.org/10.1016/j.ecolmodel.2003.09.014.

Duarte, L.O. y García, C.B. 2002. Testing responses of a tropical shelf ecosystem to fisheries management strategies. A small-scale fishery from the Colombian Caribbean Sea. En: T. Pitcher, K. Cochrane Editores. The use of ecosystem models to investigate multispecies management strategies for capture fisheries. Fisheries Centre, University of British Columbia, British Columbia.

Duarte, L. O. y C.B. García, C. B. 2001. Explorando los efectos de la expansión del área de pesca en un sistema costero tropical mediante un modelo dinámico de 
simulación espacial. Memorias IX Congreso Latinoamericano sobre Ciencias del Mar. Asociación Latinoamericana de Investigadores en Ciencias del Mar, Instituto de Estudios Caribeños, Universidad Nacional de Colombia. CD- ROM.

Dunning, J.B., Steward, D.J., Danielson, B.J., Noon, B.R., Root, T.L., Lamberson, R.H. y Stevens, E.E. 1995. Spatially explicit population models: current forms and future uses. Ecological Applications 5:3-11. Doi: https://doi.org/10.2307/1942045.

FAO. 1997. The state of world fisheries and aquaculture 1996. FAO, Rome. En: Fisher, W. Editores. 1978. FAO species Identification sheets for fishery purposes. Western Central Atlantic (fishing area 31). FAO, Roma.

Fisher, C.D. 1978. The effects of personal control, competence, and extrinsic reward systems on intrinsic motivation. Organizational Behavior \& Human Performance, 21(3): 273-288. https://doi.org/10.1016/0030-5073(78)90054-5.

Finucane, J y R. Vaught. 1986. Species profile of Atlantic thread herring, Opisthonema oglinum (Lesuer 1818). NOAA. Tech memory 182: 30.

Foote, K. 1987. Fish target strenghts for use in echo integrator surveys. The Journal of the Acoustical Society of America - Scitation 82(3): 981-987. Doi: https://doi.org/10.1121/1.395298.

Gamma Design Software, 2004. GS+: Geoestatistics for the Environmental Sciences. Gamma Designs Software, Plainwell, Michigan.

Golden Software. 2002. Surfer 8. User's guide. Golden.

Goovaerts, P. 1997. Geostatistics for Natural Resources Evaluation. Oxford University Press, New York.

Hildebrand, S. F. 1963. Familia Elopidae: En Fishes of the Western North Atlantic Memoir Sears Foundation for Marine Research 1(3): 111-131.

Holmes, E.E, Lewis, M.A., Banks, J.E. y Veit, R.R..1994. Partial differential equations in ecology: spatial interactions and population dynamics. Ecology 75:17-29. Doi: https://doi.org/10.2307/1939378.

Houde, ED., Grail, C. y Berkeley, S. 1983. Population parameter estimates for three shoaling pelagic tishes in the eastern Gulf of Mexico. Int. Count. Exploration Sea Pelagic Fish. Comm. CM 1983/H:43.

Hutchings, J.A. 2000. Collapse and recovery of marine fishes. Nature 406:882-885. Doi: https://doi.org/10.1038/35022565.

Isaaks, E.H. y Srivastava, R.M. 1989. An Introduction to Applied Geoestatistics. Oxford University Press, New York.
Jackson, J.B.C., Kirby, M.X., Berger, W.H., Bjorndal, K.A., Botsford, L.W., Bourque, B.J., Bradbury, R.H., Cooke, R., Erlandson, Estes, J. J.A., Hughes, T.P., Kidwell, S., Lange, C.B., Lenihan, H.S., Pandolfi, J.M., Peterson, C.H., Steneck, R.S., Tegner, M.J. y Warner, R.R. 2001. Historical Overfishing and the Recent Collapse of Coastal Ecosystems. Science 293: 629-637. Doi: https://doi.org/10.1126/science.1059199.

Ludwig, D., Hilborn, R. y Walters, C. 1993. Uncertainty, resource exploitation and conservation: lessons from history. Science 260: 17-36. Doi: https://doi.org/10.1126/science.260.5104.17.

Kemmerer, A. 1977. LANDSAT menhaden and thread herring resources investigation. Southeast Fisheries center Contribution 77-16; MARMAP Contribution 145.

Mangel, M. et al. 1996. Principles for the conservation of wild living resources. Ecological Applications 6(2): 338-362. Doi: https://doi.org/10.2307/2269369.

Manjarrés, L. 1993. Detección de caladeros para la pesca de recursos demersales en la Guajira. Crucero de septiembre-octubre de 1993. Boletín Técnico 01-93:1-10.

Manjarrés, L, Infante, J., Rueda, A. y Escorcia, F. 1993. Carta Pesquera del área de Santa Marta. En: Proyecto Integral de Investigaciones y Desarrollo de la Pesca Artesanal en el área Marítima de Santa Marta. Informe Técnico Final. INPA-CIID-UNIMAGDALENA, Santa Marta.

Manjarrés, L., Vergara, A., Torres, J., Rodríguez, G., Arteaga, E., Viaña, J., Arévalo, J. y Galvis, R. 2005a. Evaluación de peces demersales e ictioplancton en el Mar Caribe de Colombia, incluyendo condiciones oceanográficas. Parte I: Crucero INPA-VECEP/UE/DEMER/9507 (julio de 1995). Intropica 2: 51-86.

Manjarrés, L., Rodríguez, G., Torres, J., Vergara, A., Arteaga, E., Arévalo, J., Galvis, R. y Viaña, J., 2005b. Evaluación de peces demersales e ictioplancton en el mar Caribe de Colombia, incluyendo condiciones oceanográficas. Parte II: Crucero INPA-VECEP/UE/DEMER/9510 (octubrenoviembre de 1995). Intropica 2: 87-115.

Manjarrés, L, A. Vergara, G. Rodríguez, J. Viaña, E. Arteaga, J. Arévalo, R. Galvis, J. Rodríguez, J. Torres y M. Barros. 2005c. Evaluación de peces demersales e ictioplancton en el Mar Caribe de Colombia, incluyendo condiciones oceanográficas. Parte III: Crucero INPAVECEP/UE/DEMER/9604 (abril de 1996). Intropica 2: 117149.

Manjarrés, L.M., Mazenet, J.C., Duarte, L.O., Infante, J.H. y Cuello, F. 2004. Guía de usuario de las bases de datos pesqueros artesanales de los departamentos del Magdalena y La Guajira. Capitulo II. En: Manjarrés, L. 
Editores. Estadísticas pesqueras artesanales del Magdalena y La Guajira, con aplicación de herramientas informáticas para su sistematización y procesamiento. UNIMAGINCODER-INPA- COLCIENCIAS, Santa Marta.

Meaden, G.J. y J. Kapetsky. 1992. Los sistemas de información geográfica y telepercepción en la pesca continental y la acuicultura. FAO Documento Técnico de Pesca. Roma.

Meaden, G.J. y Do Chi, T. 1996. Geographical Information systems. Applications to marine fisheries. FAO Fisheries Technical Paper 356:1-335.

Myers, R. A. y Worm, B. 2003. Rapid worldwide depletion of predatory fish communities. Nature 423: 280-283. Doi: https://doi.org/10.1038/nature01610.

O'Neill, R.V. 2001. Is it time to bury ecosystem concept ? (With full military honors, of course). Ecology 82(12): 3275$3284 . \quad$ Doi: $\quad$ https://doi.org/10.1890/00129658(2001)082[3275:IITTBT]2.0.CO;2.

Páramo, J. y Roa, R. 2003. Acoustic-geoestatistical assessment and habitat-abundance relations of small pelagic fish from the Colombian Caribbean. Fisheries Research 60: 309-319. Doi: https://doi.org/10.1016/S01657836(02)00142-X.

Pauly, D., Christensen, V., Guenette, S., Pitcher, Sumaila T.J., Walters U.R., Watson, C.J.R. y Zeller, D. 2002. Towards sustainability in world fisheries. Nature 418:689-695. Doi: https://doi.org/10.1038/nature01017 .

Pauly, D., Alder, J., Bennett, E., Christensen, V. Tyedmers, P. y Watson, R. 2003. The future for fisheries. Science. 302: 1359-1361. Doi: https://doi.org/10.1126/science.1088667.

Petitgas, P. y Prampart, A. EVA (Estimation Variance). 1993. A geostatistical software on IBM-PC for structure characterization and variance computation. ORSTOM.

Pitcher, T. 1998. A cover story: fisheries may drive stocks to extinction. Reviews in Fish Biology and Fisheries 8: $367-$ 370. Doi: https://doi.org/10.1023/A:1008804029850.

Rivoirard, J., Simmonds, J., Foote, K.G., Fernandes, P. y Bez,
N. 2000. Geostatistics for estimating fish abundance. Blackwell Science Ltd., Londres. Doi: https://doi.org/10.1002/9780470757123.

Smith, J.W. 1994. Biology and Fishery for Atlantic Thread Herring, Opisthonema oglinum, along the North Carolina Coast. Marine Fisheries Review 56: 1-7.

Stromme, T. y G. Saetersdal. 1989. Prospecciones de los recursos pesqueros de la plataforma entre Colombia y Suriname 1988. IMR. Bergen.

Sumaila, U.R., Guenette, S., Alder, S. y Chuenpagdee R. 2000. Addressing ecosystem effects of fishing using marine protected areas. ICES Journal of Marine Science 57: 752-760. Doi: https://doi.org/10.1006/jmsc.2000.0732 .

Summers, J.K. y Rose, K.A. 1987. The role of interactions among environmental conditions in controlling historical fisheries variability. Estuaries 10(3): 255-266. Doi: https://doi.org/10.2307/1351853.

Valdés, E. y Sotolongo, M. 1983. Algunos aspectos de la biología y pesquería del machuelo (Opisthonema oglinum) de la plataforma Suroccidental cubana. Revista Cubana de Pesqueria 3: 65-67.

Vega-Cendejas, M.E., Mexicano-Cíntora, G. y Arce, A.M. 1997. Biology of the thread herring Opisthonema oglinum (Pisces: Clupeidae) from a beach seine fishery of the Campeche Bank, Mexico. Fisheries Research 30: 117-126. Doi: https://doi.org/10.1016/S0165-7836(96)00547-4 .

Viaña, J.E., Manjarrés, L.M., Rodrígue,z G. y Galvis, R. 1999. Análisis de los resultados de las campañas acústicas de evaluación de peces pelágicos pequeños en el Caribe colombiano - INPA-VECEP/UE/PELAG/97-98. Boletín Científico INPA, Suplemento especial 6: 95-108.

Walters, C., Pauly, D. y Christensen, V. 1999. Ecospace: prediction of mesoscale spatial patterns in trophic relationships of exploited ecosystems, with emphasis on the impacts of marine protected areas. Ecosystems 2: 539554. Doi: https://doi.org/10.1007/s100219900101.

Citar como: Manjarrés-Martínez, L.M. y Cuello, F. 2019. Una línea de referencia histórica acerca de la distribución espacial del machuelo (Opisthonema oglinum) en el área de afloramiento del mar Caribe de Colombia. Intropica 14(2): 148-159. Doi: http://dx.doi.org/10.21676/23897864.3390 